\title{
Pattern Recognition Receptors: Significance of Expression in the Liver
}

\author{
Jan Żeromski ${ }^{1} \cdot$ Agata Kierepa $^{2} \cdot$ Bartosz Brzezicha $^{1} \cdot$ Arleta Kowala-Piaskowska $^{2} \cdot$ Iwona Mozer-Lisewska $^{2}$
}

Received: 31 December 2019 / Accepted: 2 September 2020 / Published online: 17 September 2020

(c) The Author(s) 2020

\begin{abstract}
Pattern recognition receptors (PRRs) are a pivotal part of the immune system. They are distributed in almost every site of higher organisms, able to recognize foreign pathogens or unwanted remnants of metabolism and mount innate immune response. Moreover, PRRs create bridging signaling to initiate adaptive immunity. The liver being the largest organ of the body, exposed to myriads of foreign substances often being immunogenic, is well equipped with PRRs. They act as sentinels of the organ, both in health and disease. In viral hepatitis $C$ at least two of them, RIG-1 and TLR3 sense HCV, induce protective interferon production and create proinflammatory status. The hepatitis B virus is apparently invisible to PRRs, which has recently been denied. Besides, they are active in the course of infection. In liver injury and hepatic fibrogenesis Toll-like receptors (TLRs), predominantly TLR4, TLR3 and TLR9 are associated with gut microflora-related products and DNA from dying hepatocytes, lead to the activation of hepatic stellate cells. The latter initiate production of fibrillar collagens, the main agents forming hepatic fibrosis. Tumor cells of primary liver cancer also express PRRs, mainly TLRs. In concert with non-resolving liver inflammation, they are considered pivotal factors leading to carcinogenesis.
\end{abstract}

Keywords PRRs $\cdot$ TLRs $\cdot$ Liver $\cdot$ Hepatitis $\cdot$ Hepatic fibrosis $\cdot$ Hepatocarcinogenesis

$\begin{array}{ll}\text { Abbreviations } \\ \text { PRR } & \text { Pattern recognition receptor } \\ \text { RIG-1 } & \text { Retinoic acid-inducible gene 1 } \\ \text { TLR } & \text { Toll-like receptor } \\ \text { PAMP } & \begin{array}{l}\text { Pathogen-associated molecular pattern } \\ \text { DAMP }\end{array} \\ \text { Danger-associated molecular pattern } \\ \text { NF-kB } & \begin{array}{l}\text { Nuclear factor kappa (light-chain-enhancer } \\ \text { of activated B cells) }\end{array} \\ \text { AP } & \text { Activator protein } \\ \text { IRF } & \text { Interferon regulatory factor } \\ \text { MHC } & \text { Major histocompatibility complex } \\ \text { HSC } & \text { Hepatic stellate cell } \\ \text { NLR } & \text { NOD-like receptor } \\ \text { TRIF } & \text { TIR-domain-containing adapter-inducing } \\ & \text { interferon- } \beta \\ \text { CpG-ODN } & \text { CpG oligodeoxynucleotide }\end{array}$

Jan Żeromski

jzeromski@ump.edu.pl

1 Chair of Pathomorphology and Clinical Immunology, Karol Marcinkowski University of Medical Sciences, Poznan, Poland

2 Chair and Department of Infectious Diseases, Hepatology and Acquired Immunodeficiencies, Karol Marcinkowski University of Medical Sciences, Poznan, Poland

$\begin{array}{ll}\text { pDC } & \text { Plasmacytoid dendritic cell } \\ \text { PBMC } & \text { Peripheral blood mononuclear cell } \\ \text { NK } & \text { Natural killer cell } \\ \text { Treg } & \text { Regulatory T cell } \\ \text { Th17 } & \text { T helper 17 cell } \\ \text { HCC } & \text { Hepatocellular carcinoma } \\ \text { MDA5 } & \text { Melanoma differentiation-associated protein } \\ & 5 \\ \text { IPS-1 } & \text { Interferon-beta promoter stimulator 1 } \\ \text { SNP } & \text { Single nucleotide polymorphism } \\ \text { NOD2 } & \text { Nucleotide-binding oligomerization domain } \\ & \text { containing 2 } \\ \text { MD2 } & \text { Myeloid differentiation protein 2 } \\ \text { Tak1Hep } & \text { TGF } \beta \text {-activated kinase 1 }\end{array}$

\section{Introduction}

Pattern recognition receptors (PRRs) are evolutionally very old, germ line encoded receptors expressed on cell membranes and also in the cytoplasm of several cells but predominantly on those of the immune system. They are present in most of Eukariota but absent in Prokariota, such as unicellular bacteria, protozoa, viruses and fungi (Lemaitre et al. 1996). PRRs are considered to be pivotal members of 
innate (non-specific) immunity and they form a bridging pathway between innate and adaptive immune responses (Motta et al. 2015; Schenten and Medzhitov 2011). PRRs are grouped into six families, subdivided in sensors, each able to "see" a given molecular compound. Sensors recognize so called molecular patterns of two types. The first, named PAMPs (pathogen-associated molecular patterns) sense various substances pivotal for the growth and proliferation of unicellular organisms. The second, so-called DAMPs (danger-associated molecular patterns) recognize unwanted or noxious molecules of higher multicellular organisms such as uric acid and occasional chemical compounds, for example silica. The earliest discovered and described PRRs were Toll-like receptors (TLRs) in the fruit fly (Lemaitre et al. 1996) as antifungal agents. There are at least ten TLR items in humans, but more have been found in various animals, such as mice-13. Recognition of a particular TLR by a given compound results in downstream activation of different adaptor proteins, several cytoplasmic enzymes and other molecules leading to the maturation and assembly of transcription factors such as nuclear factor (NF)- $\mathrm{\kappa B}$, activator protein (AP)-1, interferon regulating factors (IRFs) and others. The latter enter the cell nucleus and initiate transcription of several genes encoding proinflammatory cytokines, cell adhesion molecules, MHC antigens, etc. Apart from the activation of cell metabolism, several adverse phenomena may take place in a cell. These include cell apoptosis, autophagy or even pyroptosis (cell death due to excessive inflammation) (Akira et al. 2006; Banerjee and Gerondakis 2007; Medzhitov 2013). The expression of TLRs has been demonstrated as transmembrane structures or cytoplasmic ones in cells of several human internal organs, including the liver. In the latter, practically all cell types have been found to express both RNA and protein of most known PRRs. As expected, Kupffer cells, due to their broad cell-intrinsic innate functions, have the highest expression of PRRs. Others, such as hepatocytes, hepatic stellate cells (HSC) or liver sinusoidal endothelial cells are also positive but to a lesser degree. Cells express most frequently not only TLRs, but also RIG1-like ones, DNA cytosolic sensors as well as NOD-like receptors (NLRs) (Alegre et al. 2017; Faure-Dupuy et al. 2018; Kiziltas 2016).

Apart from the significance of PRRs in various pathological conditions of the liver described below, there is a growing interest in the role of these receptors in a normal organ. For example, Lang et al. (2006) demonstrated on the example of animal experiments (mice) that the immunoprivileged status of the liver is under control of TLR3 signaling. TLR 3 activation-induced interferon (IFN)- $\alpha$ and tumor necrosis factor (TNF)- $\alpha$ release, which subsequently resulted in expression of hepatic CXCL9 chemokine. The latter enhanced $\mathrm{CD}^{+} \mathrm{T}$ cell infiltration and liver injury in mice.

\section{Viral Hepatitis}

\section{Hepatitis C}

Expression of PRRs in hepatitis B and C was noticed by several authors, including ourselves (Mozer-Lisewska et al. 2010). Two PRRs RIG-1 and TLR3 are considered crucial to establish antiviral conditions in an organism infected with hepatitis $\mathrm{C}$ virus (HCV). They both sense double-stranded RNA, essential in the HCV replication cycle, activate transcription factors IRF3 and IRF7, which results in the synthesis of type IFNs anti-HCV agents. HCV reduces TRIF, a critical TLR3 adaptor, which hampers the IFN antiviral effect (Wang et al. 2009). Imran et al. (2012) searched the data from PubMed and Google Scholar concerning the effect of the HCV proteins on PRRs in the liver. They found that some of these proteins exert negative impact on PRRs pathways, helping to evade host immune response. For example, core monomeric protein stimulates the TLR2 pathway, helping the virus to evade host innate immunity. NS3/4A damage TLR3 and RIG-1 pathways. NS5A decrease expression NKG2D on natural killer (NK) cells via the TLR4 pathway, which results in the impairment of the functioning of these cells. The HCN NS3/4A protease was also revealed to cause specific proteolysis of adaptor protein TRIF linking TLR3, which impairs the signaling of the TLR3 pathway, promoting persistent infections ( $\mathrm{Li}$ et al. 2005). These data unequivocally show direct interactions between the $\mathrm{HCV}$ proteins and the PRRs one (Fig. 1).

Interesting data have been demonstrated in relation to the interaction between plasmacytoid dendritic cells (pDCs) and C virus-infected hepatocytes. It was shown that type I IFN production is only made via TLR7 signaling, when both cell types are in direct contact but pDCs do not become infected, in spite of apparent infusion of viral RNA. Moreover, IFN production requires the concordance of cell species (human/human) and highly differentiated hepatocytes. It was concluded by the authors that this phenomenon constitutes a novel cell-cell viral RNA transfer process, which might have an important role in the course and treatment of $\mathrm{HCV}$-infected patients (Takahashi et al. 2010).

In the chronic stage of this disease at least six TLRs mRNA levels assessed by real time PCR were substantially upregulated in monocytes and $\mathrm{T}$ cells as compared to controls. Upregulation was somewhat selective because TLR4 was only increased in T cells, while TLR5 exclusively in monocytes. One explanation of this phenomenon provided by the authors was the possible innate recognition of pathogens and an attempt to mount protective immune response by HCV-bearing individuals (Dolganiuc et al. 


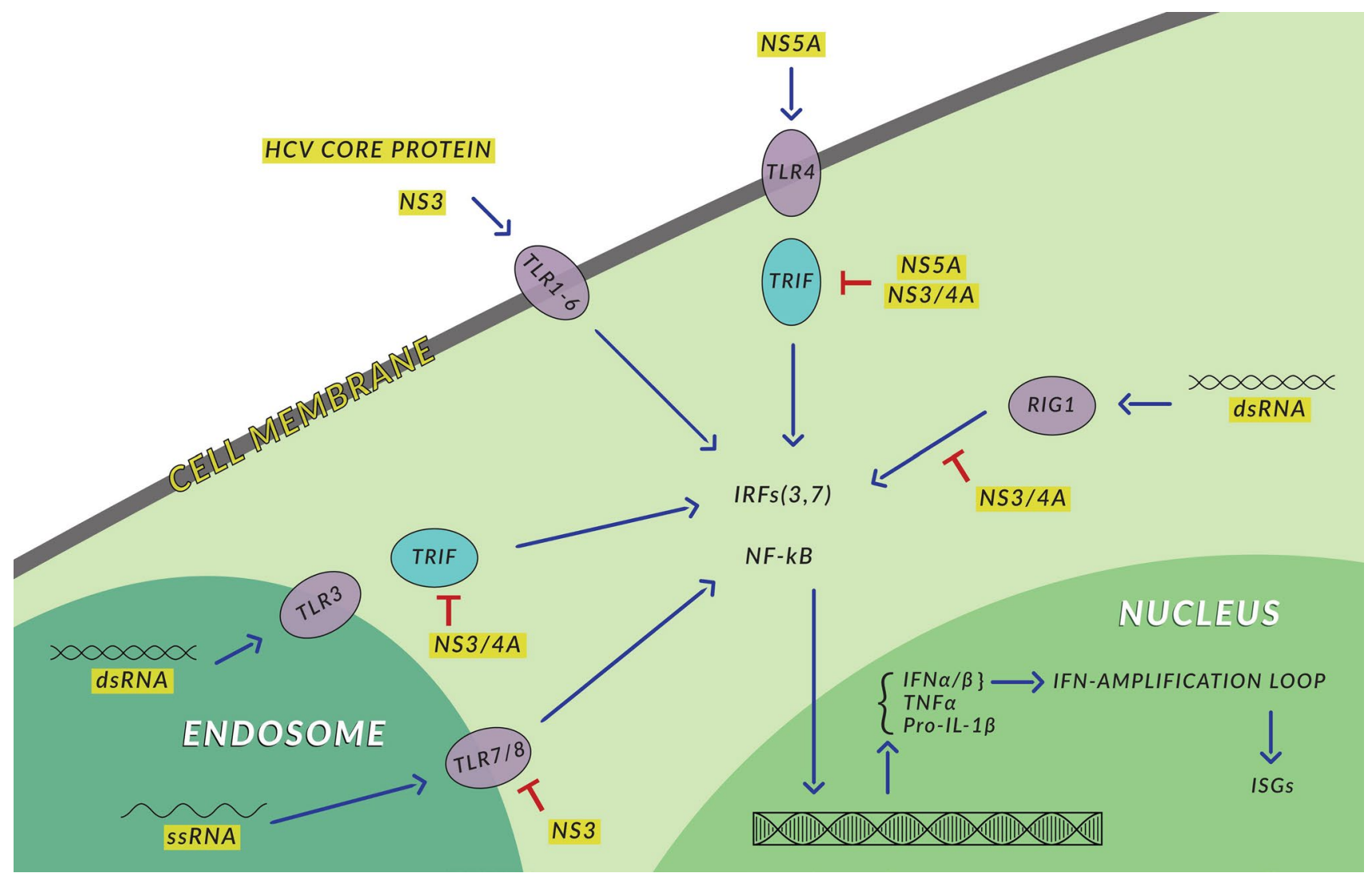

Fig. 1 Hepatitis C. Inhibition of protective immune responses in hepatocyte by viral proteins. $d s R N A$ double-stranded RNA, ssRNA single stranded RNA. Details in the text

2006). Previously, these authors indicated that the HCV core and the NS3 proteins triggered in monocytes several proinflammatory agents, such as interleukin (IL)-1 receptor-associated kinase, extracellularly regulated and c-jun $\mathrm{N}$-terminal kinases and AP-1 via TLR2 in mice. However, it was not the case in the TLR2 knockout animals (Dolganiuc et al. 2004). On the other hand, Motavaf et al. (2014) by means of sensitive DNA-based technology found significantly lower expression of TLR3 and TLR7 in peripheral blood mononuclear cell (PBMC) of 25 patients with chronic HCV infection as compared to healthy controls. It was of interest, because both mentioned TLR members recognize viral RNA in $\mathrm{PBMC}_{\mathrm{R}} \mathrm{HCV}^{+}$patients. The low expression of those TLRs may reflect the way of viral evasion of host innate immunity.

Homozygous TLR genotype association appears to be protective in chronic HCV. Mosaad et al. (2019) showed that the heterozygous CT genotype of TLR3rs 3775290 appeared to be a susceptibility risk factor of chronic HCV infection, while the homozygous CC and of TLR7rs 179008, TLR9rs 352140 single nucleotide polymorphism (SNPs), including the combined genotype (CC-AT-GA) were protective. Earlier, another group of authors (Hamdy et al. 2018) found that the TLR3rs 3775290, "CC" genotype was associated with chronic HCV infection, this SNP, however, did not correlate with the HCV RNA load of the chronic subjects. These studies indicate the potential value of genetic exploration of TLRs in this disease, in spite of evident discrepancies between individual reports.

Another group of Egyptian investigators (Mohamed et al. 2017) demonstrated links between the TLR4 expression and liver fibrosis in chronic HCV patients. In the cohort of 50 patients, fibrosis progression was positively associated with TLR4 expression. The latter was also positively correlated with several other parameters such as age, fibrosis stage, HCV RNA, serum transaminases, total bilirubin and prothrombin time but negatively with platelet count and serum albumin. The authors suggested that anti-TLR4 antibodies might limit the progression of fibrosis.

Some TLRs agonists were tested as potential adjuncts of anti-HCV therapy. As early as in 2009, Libri et al. (2009) found that out of four prototypic synthetic agonists of TLR7 or TLR9, only class C CpG oligodeoxynucleotide (CpGODN), TLR9 agonist was able to induce a robust IFN- $\alpha$ secretion by pDCs in patients with chronic HCV infection. Other proinflammatory cytokines production by infected 
pDC in response to all four agonists did not differ from the uninfected ones. It suggested a selective defect in IFN- $\alpha$ production by $\mathrm{pDC}$ from chronic HCV patients. In another study, Yuki et al. (2010) showed that low TLR3 expression was associated with good response to the treatment by peginterferon plus ribavirin in genotype 1 patients.

The impact of the direct acting antivirals (DAA) therapy on regulatory $\mathrm{T}$ cells (Tregs) and $\mathrm{T}$ helper (Th) 17 cell response to TLR2 agonist Pam3Csk4 in treated HCV patients were researched (Wei et al. 2018). The treatment resulted in the downregulated responsiveness of the abovementioned cells to TLR2 stimulation, which was interpreted as a novel mechanism of DAA-induced immunoregulation.

\section{Hepatitis B}

The situation is distinct in the case of hepatitis B virus (HBV). The virus is apparently "invisible" to the innate immune system, which was proven in both animals and human (Suslov et al. 2018). HBV-infected liver cells had the same expression of IFN or IFN stimulating genes as cells from non-infected controls. These stealth properties of the HBV are apparently not associated with the infected liver, because the IFN- $\alpha$ response could be induced by other means, such as the Sendai virus infection. The authors concluded that HBV is "invisible" to PRRs. It has, however, been recently shown that the HBV is able to induce innate immune responses in primary human hepatocytes. Following exposure to the HBV particles, hepatocytes demonstrated nuclear translocation of NF- $\mathrm{KB}$, the secretion of proinflammatory cytokines, such as IL-1 $\beta$, IL-6, TNF. The HBVinduced gene expression profile was comparable to that of a TLR2 ligand (Pam3Cys) but distinct from those known as viral sensors, TLR3 or TLR7/9. These findings contrast with the abovementioned stealth properties of HBV (Zhang et al. 2020). On the other hand, Guo et al. (2009) studied the effect of the PRRs adaptors (TRIF, RIG-1/MDA5, IPS-1) on $\mathrm{HBV}^{+}$co-transfected cells (HepG2 and Huh7). It was shown that each of the three adaptors markedly reduced the levels of HBV mRNA and DNA in both cell types. Studies on TLRs polymorphisms in HBV infection also did not exclude the role of interactions of viral molecules with TLRs in disease. The TT genotype of the missense variant TLR5 was found to be significantly more frequent in the control group than in HBV patients, indicating the protective effect of this abnormality against infection (Katrinli et al. 2018). Similar findings were also noticed for the TLR 3 gene (EzzEI-Din et al. 2017). The authors compared six SNPs in chronic HBV carriers and HBV-immunized individuals. The latter showed high frequencies of selected haplotypes (GCT CCA and CCA) as compared to chronic HBV groups. Askari et al. (2016) and Al-Anazi et al. (2019) studied nucleotide oligomerization domain 2 (NOD2) of NLRs and myeloid differentiation protein 2 (MD2), co-receptor for TLR4, polymorphisms in HBV-infected patients. They found that homozygosity of selected haplotypes of both, NOD2 gene and MD2-gene were significantly associated with HBV infection. (Boni et al. 2018) studied the TLR7 agonist (GS 9620 nucleoside analogue) in $28 \mathrm{HBV}$ patients as the therapy of HBV infection. They found greater responses to HBV and increased activation of $\mathrm{T}$ cells, manifested by higher levels of produced cytokines than in the treatment-naive group. NK cell activation and function were also increased. A 12-week therapy did not have major impact on serum hepatitis B surface antigen levels and did not significantly increase T-cell and NK-cell responses. The authors concluded that GS 9620 might be possibly applied in the therapy of HBV infection to increase specific cell-mediated immune responses.

\section{Liver Fibrosis}

Liver fibrosis is a consequence of most hepatic pathologic disorders such as hepatitis, alcoholic liver disease, or fatty liver. Chronic hepatocellular injury followed by inflammatory response results in the production of several proinflammatory and/or immunosuppressive cytokines and accumulation of the variety of inflammatory cells. This inflammatory microenvironment activates HSC, which initiate the production of several extracellular matrix proteins, such as various subtypes of collagen. This leads to the deposition of connective tissue among hepatocytes, which leads to liver fibrosis and eventually to its more severe form, namely cirrhosis. Fibrogenic stimuli acting on HSC include TGF- $\beta$, platelet-derived growth factor, proinflammatory cytokines produced by Kupffer cells and others (Friedman 2008). Human HSC express TLR4 and its co-receptors MD2 and CD14 (Paik et al. 2003). TLR4 was found to upregulate several chemokines, such as MCP, RANTES, IP10 and to inhibit Bambi-type I TGF- $\beta$ receptor but to be suppressant of TGF- $\beta$, activin and bone morphogenetic protein. Inhibition of Bambi by TLR4 enhances TGF- $\beta$ signaling in HSC and thus has promoting effect on the augmentation of liver fibrosis (Guo and Friedman 2010). It is suggested that high levels of Bambi expression inhibit TGF- $\beta$ signaling in HSC of normal livers (Aoyama et al. 2010) Apart from TLR4, HSC express also TLR2, TLR3 and TLR9. All upregulate HSC and TLR9 does it under the influence of host-derived denatured DNA from apoptotic hepatocytes (Fig. 2). Thus, TLR9 expressed on HSCs recognizing CpG-rich DNA seems to be another profibrotic agent. This leads to the differentiation of HSC and inhibition of their chemotaxis (Watanabe et al. 2007). Kupffer cells also participate in the formation of liver fibrosis through the activation of TLRs by lipopolysaccharides. It results in the production of several cytokines, including fibrogenic ones. The most significant of them all 
Fig. 2 Factors leading to hepatic fibrosis. $P D G F$ platelet-derived growth factor, $K C$ Kupffer cell. Details in the text

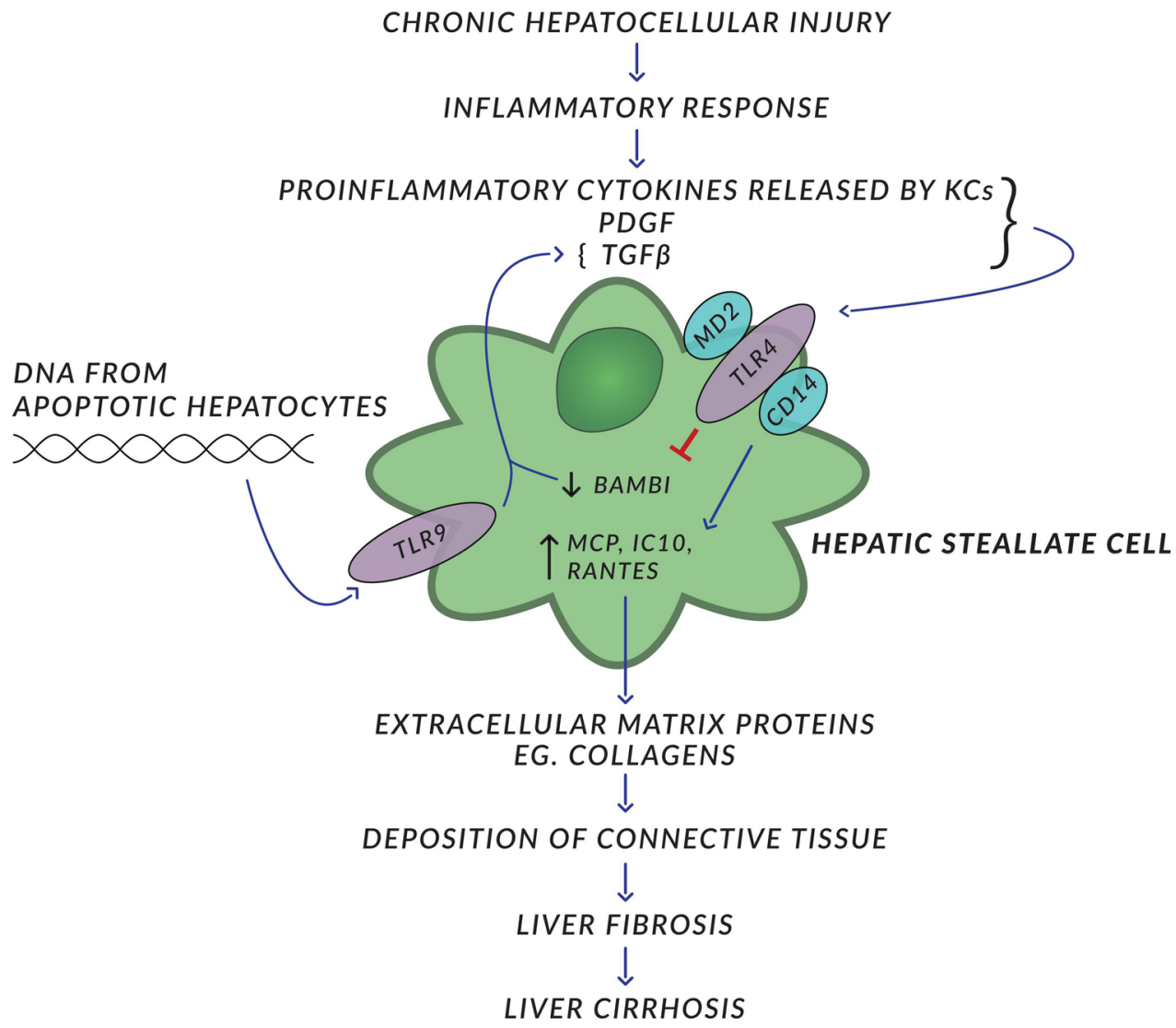

appears to be transforming growth factor (TGF)- $\beta$, binding receptor (TGF- $\beta$ R1) on HSCs, which leads to the production and release of collagen fibers (Seki et al. 2007). Recently, Chinese and American authors have reported that mice with deleted TLR4 have been protected from chronic alcoholinduced liver disease and fatty liver (Jia et al. 2018). Stimulated NK cells are cytotoxic for activated HSCs via IFN type 1. TLR3 ligand poly:IC and IFN- $\gamma$ apparently reduce liver fibrosis through TRAIL expression in NK cells (Radaeva et al. 2006). Cirrhotic individuals showed increased bacterial DNA in their plasma. Moreover, as mentioned earlier, denatured host origin DNA from dying cells stimulates HSCs via TLR9, which enhances liver fibrosis (Gabele et al. 2008).

Another group of investigators (Hartmann et al. 2012) working on genetically modified mouse models showed the crucial role of TLR2, bacteria and bacterial products translocation across the intestinal epithelial lining in concert with TNF receptor 2 signaling for the contribution to liver injury and subsequently to liver fibrosis. TNFR2 is produced by monocytes via TLR2 signaling. These data underline the role of TLRs involved in the initiation of liver fibrosis.

Apart from TLRs, another family of PRRs, namely NLR participate in liver fibrosis. Some of them are able to form bioactive protein complexes called inflammasomes, releasing proinflammatory cytokines IL- $1 \beta$ and IL-18, which initiate the activation of HSCs in chronic inflammatory liver diseases (Alegre et al. 2017; Wang et al. 2019). It has recently been reported that a selective inflammasome (NLRP3) inhibitor-MCC950 is able to alleviate liver fibrosis in mice (Ou et al. 2019).

\section{PRRs and Liver Carcinogenesis}

There are several reports, predominantly by Chinese authors but also ourselves (Żeromski et al. 2019) about the frequency of expression and significance of TLRs in hepatocellular carcinoma (HCC). For example, Sun et al. (2016) showed that about 53 and $85 \%$ of patients bearing liver cancer express TLR3 and TLR9, respectively. Tanaka et al. (2010) showed that TLR9 agonist CpG-ODN promoted HCC cell proliferation but reduced cytotoxicity of anticancer drug adriamycin. Moreover, TLR agonists closely regulated genes and transcription factors involved in tumorigenesis and progression of HCC as shown by means of gene chip microassay analysis. Non-resolving inflammation is considered an important causative factor of liver cancer (Yu et al. 2018). In mice deleted for TGF $\beta$-activated kinase 1 (Tak1Hep) known to develop inflammation and HCC, TLR4 and TLR9-MyD88 were found to be the driving forces of progression to cancer (Song et al. 2018). Zhou et al. (2019) noticed that high expression of TLR2 and TLR4 on PBMC was associated 
with intestinal overgrowth promoted the development of HCC. Another group (Hou et al. 2017) reported that the expression of hepatitis B core antigen in concert with the presence of TLR4 promoted invasion of the established HCC cell line. On the other hand, TLR3 expression on HCC cells seems to trigger cell apoptosis. Thus, Bonnin et al. (2019) concluded, based on experimental work, that TLR3 downregulation appears to be a novel escape way from apoptosis in carcinogenesis of liver. Yu et al. (2010) and later Yu et al. (2018) in a review article came to the conclusion that the activation of PRRs by PAMPs or unwanted host products from dying liver cells leading to unresolving inflammation is crucial for the induction of hepatocarcinogenesis. They even argued that the inhibition of inflammatory pathways might be a promising approach for the $\mathrm{HCC}$ prevention and therapy. They proposed the drug sorafenib as a candidate for such treatment but warned that promising experiments were only performed in mouse HCC models, which does not yet permit us to have it tested on humans. In contrast to $\mathrm{Yu}$ et al. (2018) and Dapito et al. (2012), on the basis of several highly sophisticated animal models, came to the conclusion that there is no significant contribution of intestinal microbiota and TLR4 to liver cancer induction but it is valid for the tumor promotion, mediating HCC proliferation, secretion of growth factors such as liver mitogen epiregulin and prevention of apoptosis. Nevertheless, the majority of the authors agreed that gut microflora derived from bacterial products. Some endogenous substances such as high mobility group B1 (HMGB1), free fatty acids collectively called DAMPs, in concert with TLRs, in the course of alcoholic and nonalcoholic liver disease, contribute to the hepatocarcinogenesis (Hernandez et al. 2019; Petrasek et al. 2013; Roh and Seki 2013).

\section{Other Liver Diseases}

Nonalcoholic steatohepatitis (NASH) formation and development has been found to be TLR9 pathway activation dependent. Plasma from individuals with NASH contains high amounts of highly oxidized mitochondrial DNA, mostly in microparticles from hepatocytes. This DNA, relatively $\mathrm{CpG}$ rich, is able to activate TLR9. Mitochondrial DNA acts as a ligand of TLR9, which results in the stimulation of proinflammatory cytokines in hepatic microenvironment. Total body TLR9 deletion results in the disappearance of NASH in high fat diet (HFD) fed mice. TLR9 antagonist such as IRS954 prevents NASH formation in HFD fed mice (GarciaMartinez et al. 2016).

Autoimmunity - there are reports in the literature that endogenous ligands such as nucleic acids RNA and DNA derived either from microorganisms or of mammalian origin may activate TLRs under certain conditions and initiate a typical autoimmune process such as systemic lupus erythematosus (Barrat et al. 2005; Hurst and von Landenberg 2008). Chi et al (2018) have shown in mouse models that stronger/long-lasting liver inflammation resulting from virus (adenovirus) or chemicals $\left(\mathrm{CCl}_{4}\right)$ may lead to autoimmune hepatitis by releasing the ligands for TLR2/TLR4. Upregulated cytokines and chemokines in the liver activate STAT3 and STAT4 in hepatic $\mathrm{CD} 4{ }^{+} \mathrm{CD} 25^{+}$Treg cells suppressing FOXp3 gene expression resulting in the reduction of suppressive activity of Tregs.

Malaria parasites infestation - there is a growing opinion that PRRs, mainly TLRs recognize Plasmodium components and shape innate, albeit inadequate immune response (Kalantari 2018). The group of Japanese authors (Ernest et al. 2018) reported that the TLR2 agonist (PEG-Pam2Cys) dramatically reduces the quantity of rodent malaria parasite Plasmodium yoelli that grows in mouse liver. The drug was also able to clear parasites from the liver after establishment of infection.

Gut-liver axis-bile acid metabolism connects the liver with the intestine. Dysregulation of bile acids leads to intestinal dysbiosis that promotes pathogenic microorganisms entrance in the liver via portal vein and subsequent hepatic inflammation. Dysbiosis is also due to faulty bidirectional liver-microbiome crosstalk (Adolph et al. 2018). It triggers NLRs and the formation of inflammasomes. NLRP3 inflammasome induces proinflammatory cytokine IL- $1 \beta$ that aggravates inflammatory hepatic damage and affects the epithelial integrity of cholangiocytes. Bile acids are able to activate NLRP3 inflammasome in macrophages. In contrast, the NLRP6 one maintains microbial balance and antimicrobial peptide synthesis as well as mucus secretion by goblet cells (Wang et al. 2019).

Liver transplantation-in liver transplant recipients, the damage of cells during ischemia-reperfusion injury poses a significant clinical problem. Sosa et al. (2020) found TLR4/7/9 and NOD2 expression of portal blood may be used as a marker of cell damage for prediction and/or therapeutic intervention in orthotopic liver transplant patients before transplantation.

\section{Concluding Remarks}

Pattern recognition receptors are still not fully appreciated by clinical hepatologists as an important factor in the management of liver diseases. The data provided in this article suggest that the complex nature of their function may hamper direct transfer of this knowledge to clinics. Nevertheless, the growing number of PRR synthetic ligands, showing therapeutic effect in various liver pathologies appears to be a promising agent for future liver therapies. 
Acknowledgements This work was partly supported by the National Science Centre (NCN Warsaw) under OPUS Grant 2016/23/B/ NZ6/01497 (awarded to Prof. I. Mozer-Lisewska). The text has been corrected by the native speaker Mrs. Suzann Poynton-Motylinska, reader in the Department of Foreign Languages at Poznan University of Medical Sciences.

Funding Funding was provided by Narodowe Centrum Nauki (Grant no. 2016/23/B/NZ6/01497).

\section{Compliance with Ethical Standards}

Conflict of Interest The authors declare that they have no conflict of interest.

Open Access This article is licensed under a Creative Commons Attribution 4.0 International License, which permits use, sharing, adaptation, distribution and reproduction in any medium or format, as long as you give appropriate credit to the original author(s) and the source, provide a link to the Creative Commons licence, and indicate if changes were made. The images or other third party material in this article are included in the article's Creative Commons licence, unless indicated otherwise in a credit line to the material. If material is not included in the article's Creative Commons licence and your intended use is not permitted by statutory regulation or exceeds the permitted use, you will need to obtain permission directly from the copyright holder. To view a copy of this licence, visit http://creativecommons.org/licenses/by/4.0/.

\section{References}

Adolph TE, Grander C, Moschen AR et al (2018) Liver-microbiome axis in health and disease. Rev Trends Immunol 39:712-723

Akira S, Uematsu S, Takeuchi O (2006) Pathogen recognition and innate immunity. Cell 124:783-801

Al-Anazi MR, Nazir N, Abdo AA et al (2019) Genetic variations of NOD2 and MD2 genes in hepatitis B infection. Saudi J Biol Sci 26:270-280

Alegre F, Pelegrini P, Feldstein AE (2017) Inflammasomes in liver fibrosis. Semin Liver Dis 37:119-127

Aoyama T, Paik YH, Seki E (2010) Toll-like receptor signaling and liver fibrosis. Gastroenterol Res 2010:192543

Askari AA, Nostratabadi R, Khaleghinia M et al (2016) Evaluation of NLRC4, NLRP1 and NLRP3 as components of inflammasomes, in chronic hepatitis B virus infected patients. Viral Immunol 29:496-501

Banerjee A, Gerondakis S (2007) Coordinating TLR-activated signaling pathways in cells of the immune system. Immunol Cell Biol $85: 420-424$

Barrat FJ, Meeker T, Gregorio J et al (2005) Nucleic acids of mammalian origin can act as endogenous ligands for toll-like receptors and may promote systemic lupus erythematosus. J Exp Med 202:1131-1139

Boni C, Vecchi A, Rossi M et al (2018) TLR7 agonist increases responses of hepatitis $\mathrm{B}$ virus specific T cells and natural killer cells in patients with chronic hepatitis B treated with nucleos(T) ide analogues. Gastroenterology 154:1764-1777

Bonnin M, Fares N, Testoni B et al (2019) Toll-like receptor 3 downregulation is an escape mechanism from apoptosis during hepatocarcinogenesis. J Hepatol 71:763-772

Chi G, Feng XX, Ru YX et al (2018) TLR2/4 ligand amplified liver inflammation promotes initiation of autoimmune hepatitis due to sustained IL-6/IL-12/IL-4/IL-25 expression. Mol Immunol 99:171-181

Dapito DH, Mencin A, Gwak GY et al (2012) Promotion of hepatocellular carcinoma by the intestinal microbiota and TLR4. Cancer Cell 21:504-516

Dolganiuc A, Garcia C, Kodys K et al (2006) Distinct Toll-like receptor expression in monocytes and $\mathrm{T}$ cells in chronic HCV infection. World J Gastroenterol 12:1198-1204

Dolganiuc A, Oak S, Kodys K et al (2004) Hepatitis C core and nonstructural 3 proteins trigger Toll-like receptor 2-mediated pathways and inflammatory activation. Gastroenterology 127:1513-1524

Ernest M, Hunja C, Arakura Y et al (2018) The Toll-like receptor 2 agonist PEG-Pam2Cyus as an immunochemoprophylactic and immunochemotherapeutic against the liver and transmission stages of malaria parasites. Int J Parasitol Drugs Drug Resist 8:451-458

EzzEl-Din AM, Galal SH, Ahmed AO et al (2017) Toll-like receptor 3 polymorphisms and hepatitis B virus infection. Egypt $\mathrm{J}$ Immunol 24:173-185

Faure-Dupuy S, Vegna S, Aillot L et al (2018) Characterization of pattern recognition receptor expression and functionality in liver primary cells and derived cell lines. J Innate Immunol 10:339-348

Friedman SL (2008) Mechanisms of hepatic fibrogenesis. Gastroenterology 134:1655-1669

Gabele E, Muhlbauer M, Dorn C et al (2008) Role of TLR9 in hepatic stellate cells and experimental liver fibrosis. Biochem Biophys Res Commun 376:271-276

Garcia-Martinez I, Santoro N, Chen Y et al (2016) Hepatocyte mitochondrial DNA drives nonalcocholic steatohepatitis by activation of TLR9. J Clin Investig 126:859-864

Guo H, Jiang D, Ma D et al (2009) Activation of pattern recognition receptor-mediated innate immunity inhibits the replication of hepatitis B virus in human hepatocyte-derived cells. J Virol 83:847-858

Guo J, Friedman SL (2010) Toll-like receptor 4 signaling in liver injury and hepatic fibrogenesis. Fibrogenesis Tissue Repair 3:21

Hamdy S, Osman AM, Zakaria ZA et al (2018) Association of Toll-like receptor 3 and Toll-like receptor 9 single nucleotide polymorphisms with hepatitis $\mathrm{C}$ virus persistence among Egiptians. Arch Virol 163:2433-2442

Hartmann P, Haimerl M, Mazagova M et al (2012) Toll-like receptor 2-mediated intestinal injury and enteric tumor necrosis factor receptor I contribute to liver fibrosis in mice. Gastroenterology 143:1330-1340

Hernandez C, Huebener T, Pradere JP et al (2019) HMGB1 links chronic liver injury to progenitor responses and hepatocarcinogenesis. J Clin Investig 129:1803

Hou HR, Kang Y, Li YK et al (2017) Hepatitis B core antigen promotes invasion of hepatocellular carcinoma cell line HepG2.2 via Tolllike receptor 4. Zhonghua Gan Zang Bing Za Zhi 25:908-913

Hurst J, von Landenberg P (2008) Toll-like receptors and autoimmunity. Autoimmun Rev 7:204-208

Imran M, Waheed Y, Manzoor S et al (2012) Interaction of Hepatitis $\mathrm{C}$ virus proteins with pattern recognition receptors. Virol J 9:126

Jia L, Chang X, Qian S et al (2018) Hepatocyte Toll-like receptor 4 deficiency protects against alcohol-induced fatty liver disease. Mol Metab 14:121-129

Kalantari P (2018) The emerging role of pattern recognition receptors in pathogenesis of malaria. Vaccines 6:E13

Katrinli S, Nigdelioglu A, Ozdil K et al (2018) The association of variations in TLR genes and spontaneous immune control of hepatitis B virus. Clin Res Hepatol Gastroenterol 42:139-144

Kiziltas S (2016) Toll-like receptors in pathophysiology of liver diseases. World J Hepatol 8:1354-1369 
Lang KS, Georgiev P, Recher M et al (2006) Immunoprivileged status of the liver is controlled by toll-like receptor 3 signaling. J Clin Investig 116:2456-2463

Lemaitre B, Nicolas E, Michaut L et al (1996) The dorsoventral regulatory gene cassette spatzle/Toll/cactus controls to potent antgifungal response in Drosophila adults. Cell 86:973-983

Li K, Foy E, Ferreon JC et al (2005) Immune evasion by hepatitis C virus NS3/4A protease toll-like receptor 3 adaptor protein TRIF. Proc Natl Acad Sci USA 102:2992-2997

Libri NA, Barker SJ, Rosenberg WM et al (2009) A class C CpG tolllike receptor 9 agonist successfully induces robust interferonalpha production by plasmacytoid dendritic cells from patients chronically infected with hepatitis C. J Viral Hepatol 16:315-324

Medzhitov R (2013) Pattern recognition theory and the launch of modern innate immunity. J Immunol 191:4473-4474

Mohamed SY, Mostafa EF, Hanafy AS et al (2017) The relationship between expression of Toll-like receptor 4 in chronic hepatitis $\mathrm{C}$ patients and different stages of liver fibrosis. Gastroenterol Hepatol Bed Bench 10:278-283

Mosaad YM, Metwally SS, Farag RE et al (2019) Association between Toll-like receptor 3 (TLR3) rs 3775290, TLR7rs 179008, TLR9rs 352140 and chronic HCV infection. Immunol Investig 48:321-332

Motavaf M, Noorbakhsh F, Alavian SM et al (2014) Distinct Toll-like receptor 3 and 7 expression in peripheral blood mononuclear cells from patients with chronic hepatitis $\mathrm{C}$ infection. Hepatol Mon 14:e16421

Motta V, Soares F, Sun T et al (2015) NOD-like receptors: versatile cytosolic sentinels. Physiol Rev 95:149-178

Mozer-Lisewska I, Sikora J, Kowala-Piaskowska A et al (2010) The incidence and significance of pattern recognition receptors in chronic viral hepatitis B and C in man. Arch Immunol Ther Exp 58:295-302

Ou J, Yuan Z, Wang G et al (2019) The selective NLRP3 inflammasome inhibitor MCC950 alleviates cholestatic liver injury and fibrosis in mice. Int Immunopharmacol 70:147-155

Paik YH, Schwabe RF, Bataller F et al (2003) Toll-like receptor 4 mediates inflammatory signaling by bacterial lipopolysaccharide in human hepatic stellate cells. Hepatology 37:1043-1055

Petrasek J, Csak T, Ganz M (2013) Differences in innate immune signaling between alcoholic and non-alcoholic steatohepatitis. J Gastroenterol Hepatol 28(Suppl 1):93-98

Radaeva S, Sun R, Jaruga B et al (2006) Natural killer cells ameliorate liver fibrosis by activated stellate cells in NKG2D-dependent and tumor necrosis factor-related apoptosis- inducing ligand-dependent manners. Gastroenterology 130:435-452

Roh YS, Seki E (2013) Toll-like receptors in alcoholic liver disease non-alcoholic steatohepatitis and carcinogenesis. J Gastroenterol Hepatol 28(Suppl 1):38-42

Schenten D, Medzhitov R (2011) The control of adaptive immune responses by the innate immune system. Adv Immunol 109:87-124

Seki E, De Minicis C, Osterreicher H et al (2007) TLR4 enhances TGF-beta signaling and hepatic signaling. Nat Med 13:1324-1332

Song IJ, Yang YM, Inokuchi-Shimizu S et al (2018) The contribution of toll-like receptor signaling to the development of liver fibrosis and cancer in hepatocyte-specific TAK1-deleted mice. Int J Cancer 142:81-91

Sosa RA, Rossetti M, Naini BV et al (2020) Pattern recognition receptor-reactivity screening of liver transplant patients: potential for personalized and precise organ matching to reduce risks of ischemia-reperfusion injury. Ann Surg 271:922-931

Sun L, Dai JJ, Hu WF et al (2016) Expression of toll-like receptors in hepatic cirrhosis and hepatocellular carcinoma. Genet Mol Res 15(2):3. https://doi.org/10.4238/gmr.15027419

Suslov A, Boldanova T, Wang X et al (2018) Hepatitis B virus does not interfere with innate immune responses in the human liver. Gastroenterology 154:1778-1790

Takahashi K, Asabe S, Wieland S et al (2010) Plasmacytoid dendritic cells sense hepatitis $\mathrm{C}$ virus-infected cells, produce interferon, and inhibit infection. Proc Natl Acad Sci USA 107:7431-7436

Tanaka J, Sugimoto K, Shiraki K et al (2010) Functional cell surface expression of toll-like receptor 9 promotes cell proliferation and survival in human hepatocellular carcinoma. Int J Oncol 37:805-814

Wang J, Dong R, Zheng S (2019) Role of the inflammasome in the gut-liver axis (review). Mol Med Rep 19:3-14

Wang N, Liang Y, Devaraj S et al (2009) Toll-like receptor 3 mediates establishment of antiviral state against hepatitis $\mathrm{C}$ virus in hepatoma cells. J Virol 83:9824-9834

Watanabe A, Hashmi D, Gomes A et al (2007) Apoptotic hepatocyte DNA inhibits hepatic stellate cell chemotaxis via Toll-like receptor 9. Hepatology 46:1509-1518

Wei K, Jiang BC, Guan JH et al (2018) Decreased CD4+, CD2+, CD127dim/- regulatory $T$ cells and $T$ helper 17 cell responsiveness to Toll-like receptor 2 in chronic hepatitis patients with Daclatasvir plus Asunaprevir therapy. Viral Immunol 31:559-567

Yu S, Chen J, Wu M et al (2010) Hepatitis B virus polymerase inhibits RIG-1 and Toll-like receptor 3-mediated beta interferon induction in human hepatocytes through interference with interferon regulatory factor 3 activation and dampening of the interaction between TBK1/KKepsilon and DDX3. J Gen Virol 91:2080-2090

Yu LX, Ling Y, Wang HY (2018) Role of nonresolving inflammation in hepatocellular carcinoma development and progression. NPJ Precis Oncol 2:6

Yuki N, Matsumoto S, Kato M et al (2010) Hepatic toll-like receptor 3 expression in chronic hepatitis $\mathrm{C}$ genotype 1 correlates with treatment response to peginterferon plus ribavirin. J Viral Hepatol 17:130-138

Zhang Z, Trippler M, Real C et al (2020) Hepatitis B virus particles activate Toll-like receptor 2 signaling initial upon infection of primary hepatocytes. Hepatology. https://doi.org/10.1002/hep.31112

Zhou DX, Ma YJ, Chen GY et al (2019) Relationship of TLR2 and TLR4 expressions on the surface of peripheral blood mononuclear cells to small intestinal bacteria overgrowth in patients with hepatocellular carcinoma. Zhonghua Gan Zang Bing Za Zhi 27:286-290

Żeromski J, Kaczmarek M, Boruczkowski M et al (2019) Significance and role of pattern recognition receptors in malignancy. Arch Immunol Ther Exp 67:133-141

Publisher's Note Springer Nature remains neutral with regard to jurisdictional claims in published maps and institutional affiliations. 